\title{
LA FORMACIÓN DE FORMADORES EN EDUCACIÓN SEXUAL BÁSICA
}

\author{
Fernández-García, 0. \\ Dpto de Psicología Evolutiva y de la Educación, Universitat de València, Valencia, España \\ Gil-Llario, M.D. \\ Dpto de Psicología Evolutiva y de la Educación, Universitat de València, Valencia, España \\ Ballester-Arnal, $\mathbf{R}$. \\ Dpto Psicología Básica, Clínica y Psicobiología, Universitat Jaume I, Castellón, España \\ Castro-Calvo, J. \\ Dpto Psicología Básica, Clínica y Psicobiología, Universitat Jaume I, Castellón, España \\ Dolores.Gil@uv.es \\ https://doi.org/10.17060/ijodaep.2017.n1.v3.1009
}

Fecha de Recepción: 30 Marzo 2017

Fecha de Admisión: 1 Abril 2017

\section{RESUMEN}

Introducción: La formación de los futuros educadores sexuales requiere no solo de un elevado grado de especialización, sino también del dominio de una serie de habilidades y competencias ya que precisa de una constante adaptación a las características del colectivo al que se dirige. La formación de estos educadores, más allá de cumplir el objetivo de proporcionar conocimientos específicos, debe asegurar el dominio percibido de las destrezas para llevar a cabo distintos formatos de intervención según el tamaño y características del colectivo diana así como del tiempo y los objetivos perseguidos. Para lograr estos objetivos, se ha ideado una propuesta de trabajo que proporciona experiencias variadas rentabilizando al máximo el tiempo lectivo. Método: En esta experiencia participaron 25 estudiantes matriculados en la asignatura "Prevención de prácticas de sexo inseguras" del máster oficial en Intervención Psicológica en el Ámbito Social de la Universitat de València, a los que se les administró tanto al inicio como al final del curso un instrumento elaborado ad hoc que evaluaba el grado de conocimientos, autoeficacia y percepción de competencia en el ámbito de la educación afectivo-sexual. Resultados: La propuesta ha sido valorada positivamente no solo en términos de incremento de conocimientos y habilidades, sino también y sobre todo en cuanto a percepción de autoeficacia y competencia de los futuros educadores. Así, el porcentaje de los que tras el curso consideraban que contaban con conocimientos suficientes como para diseñar de forma autónoma un programa de educación afectivo-sexual y que contaban con estrategias para su implementación fue del $72 \%$ y el $60 \%$ respectivamente (frente al $0 \%$ y el $12 \%$ inicial). Conclusiones: Esta propuesta metodológica de formación de formadores se ha mostrado eficaz a la hora de incrementar no sólo los conocimientos teóricos acerca del diseño de programas de intervención afectivo- 


\section{LA FORMACIÓN DE FORMADORES EN EDUCACIÓN SEXUAL BÁSICA}

sexuales, sino también la percepción de autoeficacia en su implementación en cualquier colectivo y contexto.

Palabras clave: educadores afectivo-sexuales; propuesta metodológica; formación de educadores; prevención

\section{ABSTRACT}

Training of trainers in basic sex education

Introduction: The training of future sexual educators requires not only a high degree of specialization, but also the mastery of a series of skills and competences since it requires a constant adaptation to the characteristics of the target group. The training of these educators, beyond fulfilling the objective of providing specific knowledge, must ensure the perceived mastery of the skills to carry out different intervention formats according to the size and characteristics of the target group as well as the time and the objectives pursued. In order to achieve these objectives, a work proposal has been devised that provides varied experiences, maximizing the teaching time. Method: In this experiment 25 students enrolled in the subject "Prevention of unsafe sex practices" of the official master's degree in Psychological Intervention in the Social Area of the University of Valencia, who were administered both at the beginning and at the end of the course An instrument developed ad hoc that evaluated the degree of knowledge, self-efficacy and perception of competence in the field of affective-sexual education. Results: The proposal has been positively valued not only in terms of increasing knowledge and skills, but also and especially in terms of perception of self-efficacy and competence of future educators. Thus, the percentage of those who after the course considered that they had enough knowledge to design an autonomous program of affective-sexual education and that had strategies for their implementation was $72 \%$ and $60 \%$ respectively (compared to $0 \%$ and the initial 12\%). Conclusions: This methodological proposal of training of trainers has proved effective in increasing not only the theoretical knowledge about the design of programs of affective-sexual intervention, but also the perception of self-efficacy in its implementation in any group and context.

Key words: Affective-sexual education; Methodological proposal; Training of educators; Prevention

\section{INTRODUCCIÓN:}

La adecuada formación de los educadores sexuales es objeto de primer orden ya que es necesario que se conviertan en fuentes confiables de conocimientos y habilidades (Stirling, Rees, Kasedde \& Hankins, 2008). En este sentido, la formación de los futuros educadores sexuales precisa no solo de la adquisición de un elevado nivel de especialización en el área, sino también de actitudes y habilidades con las que poder facilitar el proceso de adquisición de competencias que deben realizar los educandos. La especialización en educación sexual, por tanto, más allá de su función de actualización de conocimientos debe asegurar el dominio de las destrezas necesarias para hacer que los profesionales puedan iniciar su desempeño profesional sintiéndose lo suficientemente autoeficaces como para poner en práctica lo aprendido.

Cuando hablamos de competencia hacemos referencia a una combinación de destrezas, conocimientos, aptitudes y actitudes que incluyen la disposición para aprender, la capacitación efectiva y permiten realizar adecuadamente una tarea, función o rol (Comisión Europea, 2004; Ribes, 2006). Concretamente el concepto de competencia profesional, de naturaleza multidimensional, incluye diferentes niveles en su definición y hace referencia a la habilidad para utilizar eficazmente los recursos teóricos, técnicos y procedimentales adquiridos, así como los conocimientos y capacidades que el ejercicio de una profesión exige en cada área de intervención. Integra dos tipos de saberes: cono- 
cimientos de tipo declarativo, relacionados con el saber qué, y conocimientos procedimentales, vinculados con el saber hacery con el ejercicio de destrezas y habilidades para resolver problemas concretos (Piña, 2010; Sánchez, Castañeiras y Posada, 2012).

La autoeficacia, por su parte, hace referencia a los juicios que cada persona realiza acerca de su capacidad. Dichos juicios influyen en el modo en que organiza y actúa con objeto de alcanzar el rendimiento deseado. La autoeficacia ejerce un papel central en la autoevaluación de las capacidades personales relacionadas con la competencia profesional y constituye un importante predictor del desempeño (Bandura, 1987). Comprende el conjunto de creencias sobre los propios recursos y la eficacia personal para manejar las demandas y desafíos derivados de un área de actividad específica (Bandura, 1997; Sanjuán, Pérez \& Bermúdez, 2000). De hecho, la autoevaluación de las propias capacidades, recursos y competencias afecta la motivación y el comportamiento, ya que un desempeño óptimo no sólo se definirá por disponer de las habilidades necesarias sino también por la autoeficacia percibida para utilizar dichos recursos.

Estos son los principios que rigen la formación en educación afectivo-sexual de los futuros profesionales que cursan el Máster oficial en Intervención Psicológica en el Ámbito Social (Universitat de València). En el momento en el que se planteó la inclusión de estos contenidos, los alumnos presentaban bajos niveles de conocimientos básicos relativos a sexualidad (desarrollo del género, orientación sexual, respuesta sexual, comportamientos de riesgo, etc.), de modo que una de las principales tareas radicaba en cubrir ese vacío de conocimientos declarativos. Por otro lado, poseían algunas directrices sobre cómo elaborar programas de prevención/intervención en el ámbito de la salud, pero no habían tenido oportunidad de poner en práctica habilidades en situaciones reales. Así, resultaba esencial proporcionar un amplio abanico de casos prácticos que les permitieran experimentar directa o indirectamente cómo debía llevarse a cabo el diseño de talleres afectivo-sexuales orientados hacia un colectivo. Por ello, se ideó una propuesta educativa que proporcionara experiencias variadas rentabilizando al máximo el tiempo lectivo. Esta propuesta de trabajo tenía como objetivo asentar los conocimientos sobre sexualidad y proporcionar herramientas para un aprendizaje autónomo, a través del diseño de situaciones de enseñanza/aprendizaje en diferentes contextos.

En concreto, esta propuesta se abordó en la asignatura de "Prevención de prácticas de sexo inseguras". En la actualidad, esta asignatura se organiza en torno a tres bloques. El primer bloque tiene como meta proporcionar al estudiante una formación teórica básica sobre sexualidad en la que se analizan las dimensiones de la sexualidad y los cambios que se van produciendo a lo largo de todo el ciclo vital para sentar las bases de la educación afectivo-sexual.

En el segundo bloque se proporciona una formación especializada en la que se analizan las particularidades de los distintos colectivos, identificando la información clave que permite diseñar programas de prevención y/o de intervención en contextos específicos y adaptando los diferentes recursos en función del colectivo, de los objetivos y de la temporalización. Los colectivos a quienes se dirige la educación afectivo-sexual son: adolescentes, jóvenes en riesgo social, padres, discapacitados intelectuales y/o físicos, personas mayores, profesionales del sexo, personas con enfermedades que afectan su salud sexual (p.e., enfermos renales), etc. Los objetivos se enmarcan en la prevención/intervención en problemas causados 0 exacerbados por factores de tipo social y/o que tienen consecuencias en la salud comunitaria. Éstos varían según la etapa del desarrollo psicosexual del colectivo al que se dirijan, sus circunstancias sociales, etc.

En el tercer bloque se desarrollan las destrezas necesarias para ser un buen educador sexual. Para ello, los estudiantes diseñan por grupos programas de prevención y/o de intervención según una serie de supuestos, aplicando los contenidos y directrices desarrolladas a lo largo de los blo- 


\section{LA FORMACIÓN DE FORMADORES EN EDUCACIÓN SEXUAL BÁSICA}

ques anteriores, y posteriormente los ponen en práctica a través de role-playing. La formación se completa con un debate cuya finalidad es perfeccionar las competencias tanto relativas al manejo del grupo como a la selección de materiales, grado de adecuación de la actividad, etc.

El objetivo del presente trabajo fue evaluar la eficacia de esta breve intervención a la hora de: 1) mejorar los conocimientos acerca de sexualidad en profesionales que en un futuro pudieran dedicarse a la actividad profesional en este ámbito; 2) mejorar su percepción de eficacia a la hora de diseñar e implementar programas y talleres de educación afectivo-sexual y; 3) dotarles de recursos y habilidades de afrontamiento en el caso de que durante el transcurso de su actividad profesional, debieran enfrentarse a problemas inesperados. De constatarse su eficacia, dispondríamos de un programa de formación breve que cubriría eficazmente uno de los grandes vacíos actuales en la formación de los profesionales de la salud: el de la atención a la salud sexual.

\section{MÉTODO:}

\subsection{Participantes:}

En el estudio participaron 25 estudiantes matriculados en la asignatura "Prevención de prácticas de sexo inseguras" del máster oficial en Intervención Psicológica en el Ámbito Social de la Universitat de València. La media de edad fue de 24,44 años, estando el rango entre los 23 y los 28 años (el 48\% tenían 24 años). Respecto al sexo, 24 fueron chicas (96\%). En el momento de la realización del master, ninguno de ellos estaba trabajando y el 78\% habían terminado la carrera el curso anterior.

\subsection{Medidas:}

La escala usada para la evaluación de los participantes al inicio y al final de la asignatura fue un instrumento elaborado ad hoc que evaluaba el grado de conocimientos, la autoeficacia y la percepción de competencia en el ámbito de la educación afectivo-sexual (Anexo I). Se trata de un instrumento breve de 4 ítems con tres alternativas de respuesta elaborado sobre la base de la Escala de Autoeficacia General (Sanjuán, Pérez \& Bermúdez, 2000) ajustándola a las características de la formación de educadores sexuales.

\subsection{Procedimiento:}

Como hemos comentado anteriormente, dicha experiencia educativa se enmarca en el contexto de una asignatura de especialización en educación sexual básica del Máster oficial de Intervención Psicológica en el Ámbito Social.

En primer lugar, durante la sesión con la que se da inicio al curso, los alumnos recibieron información sobre cómo se estructuraba la asignatura (3 bloques) y qué metodología seguía. Tras ello, antes de empezar con el desarrollo de los bloques, se les administró la escala de percepción de autoeficacia y competencia como educador sexual.

Durante las 20 horas siguientes se llevó a cabo el desarrollo de los 3 bloques comentados anteriormente. En primer lugar, se proporcionaron conocimientos declarativos sobre sexualidad (5 horas); en segundo lugar, se facilitó información sobre los distintos colectivos y contextos clave a la hora desarrollar programas de prevención/intervención (5 horas); y en tercer lugar, se llevó a cabo el diseño y desarrollo de un programa inscrito dentro de un marco concreto de prevención/intervención.

Una vez concluido el curso y tras trabajar los 3 bloques, se volvieron a evaluar los conocimientos y competencias de los participantes mediante el mismo instrumento ad hoc. Esto nos permite comprobar la eficacia tanto de la selección de objetivos como de la metodología instruccional. 


\section{RESULTADOS:}

A continuación (figuras de la 1 a la 4) se muestran los porcentajes de respuesta de los participantes en la escala de percepción de autoeficacia y competencia como educador sexual antes del inicio del curso y una vez concluida la evaluación del mismo.

\section{Figura 1.}

Porcentaje de respuesta a la pregunta: ¿Qué harías si te piden que diseñes un programa de educación afectivo sexual para un colectivo con el que nunca has trabajado?

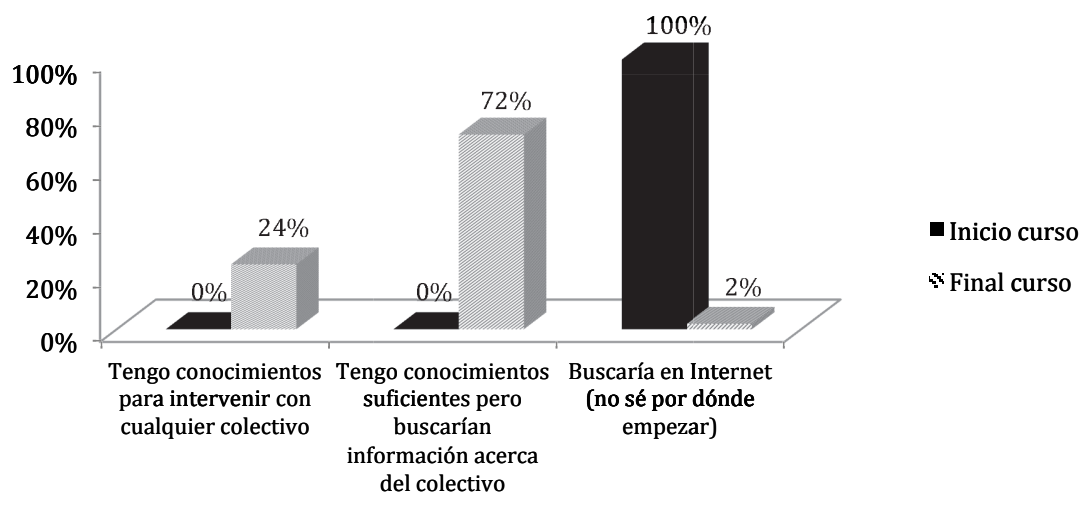

Como se aprecia en la figura 1, antes del inicio del curso todos ellos habrían recurrido a Internet en el supuesto de que les hubieran pedido diseñar un programa de educación afectivo-sexual para un colectivo con el que nunca hubieran trabajado. Sin embargo, tan sólo un $2 \%$ emplearía esta estrategia tras la finalización del curso; en su lugar, el $24 \%$ creía que poseía conocimientos suficientes para intervenir en cualquier colectivo y el $72 \%$ que los poseía y tan sólo debería ajustarlos al colectivo objeto de la intervención.

Figura 2.

Porcentaje de respuesta a la pregunta: ¿Qué harías si te piden que prepares un taller, escogiendo y/o creando actividades para un colectivo con el que nunca has trabajado?

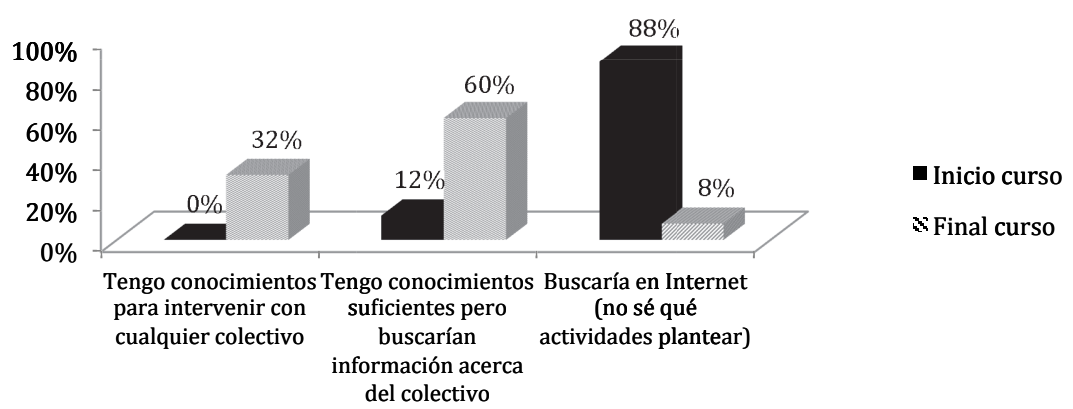




\section{LA FORMACIÓN DE FORMADORES EN EDUCACIÓN SEXUAL BÁSICA}

La tendencia sería muy similar en caso de que se les hubiera planteado desarrollar actividades concretas para un taller con un colectivo con el que nunca hubieran trabajado (figura 2). En este sentido, antes del curso el 88\% habría recurrido a Internet a la hora de escoger las dinámicas a trabajar. Una vez realizado, la mayoría (el $60 \%$ ) consideró que disponía de los conocimientos y que les bastaría con recabar un poco más de información sobre el colectivo concreto.

Figura 3.

Porcentaje de respuesta a la pregunta: ¿Cómo reaccionarían si durante un taller te encuentras con alguna dificultad?



Cuando se les preguntó acerca de cómo creían que sería su reacción ante un imprevisto durante la impartición de dichos talleres (figura 3), antes del curso el $72 \%$ afirmaba que no habría sabido reaccionar. Tras el curso, aumentó hasta el $44 \%$ y el $32 \%$ el porcentaje de los que creían que podrían hacerle frente dado que ya disponían de estrategias de afrontamiento y de los que se bloquearían inicialmente pero que después sabrían qué hacer, respectivamente.

Figura 4.

Porcentaje de respuesta a la pregunta: ¿Qué harías si te dicen que debes trabajar con un grupo difícil?

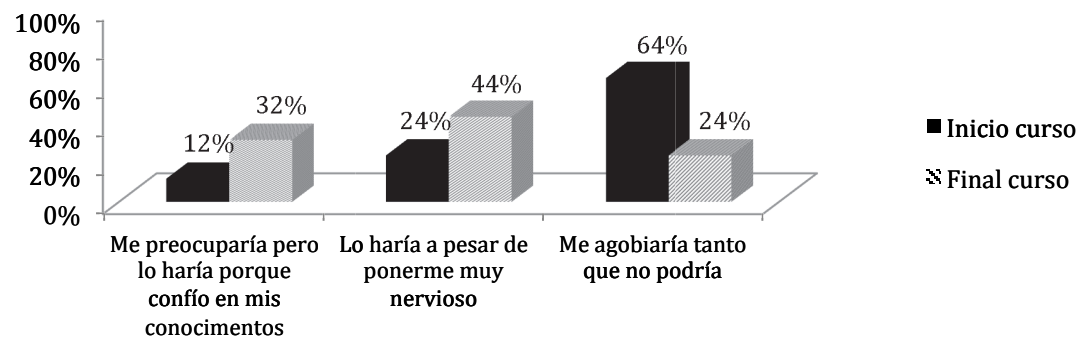

Para terminar, se les preguntó acerca de su reacción si les dijeran que debían trabajar con un grupo difícil (figura 4). Antes del curso, el $64 \%$ afirmaba que se agobiaría tanto que no se vería capaz de afrontarlo mientras que después del curso, tan sólo el 24\% experimentaría esta reacción. En su lugar, el $32 \%$ se preocuparía pero lo afrontaría porque confiaba en sus conocimientos y destrezas y el $44 \%$ lo haría aunque se pondría muy nervioso/a.

\section{DISCUSIÓN:}

El principal objetivo de este estudio ha sido probar la eficacia de una experiencia educativa innovadora con la que se buscaba lograr una mayor sensación de competencia y autoeficacia al propor- 
cionar herramientas para fomentar la salud sexual mediante el diseño e implementación de programas de educación afectivo-sexual en diferentes contextos. Para valorar la eficacia de dicha propuesta de trabajo, se evaluó la percepción de competencia y autoeficacia de los participantes antes del inicio del curso y una vez concluida la evaluación del mismo.

Antes de iniciar el curso, los estudiantes presentaban una baja percepción de autoeficacia, ya que señalaban que no contaban con suficientes conocimientos y habilidades como para diseñar y aplicar un programa de educación afectivo-sexual. En este sentido, todos los participantes indicaron que buscarían en Internet programas hechos porque no sabrían por dónde empezar, no habiendo ninguna persona que creyera poseer suficientes conocimientos teóricos ni suficientes estrategias para manejar problemas prácticos. Sin embargo, después de una formación de menos de 30 horas, se ha conseguido obtener una mejora en la percepción de eficacia que se traduce en un porcentaje del $72 \%$ que consideraba que tras esta breve instrucción, poseía suficientes conocimientos y estrategias generales. Respecto a esto, cabe matizar que el bloque dedicado a proporcionar conocimientos fue relativamente breve, consistiendo no tanto en facilitar conocimientos nuevos sino más bien en actualizar información que en realidad sí poseían pero de la que no eran conscientes. En este sentido, se comprueba que no basta con poseer información, es necesario "percibir" que se posee, siendo muy importantes las experiencias educativas útiles y reales, y en este sentido, este curso ha cumplido con dicho objetivo.

Por otro lado, también se han observado cambios respecto a la percepción de eficacia en cuanto a las habilidades. Aquí la mejora también ha sido evidente ya que al finalizar el curso, un $60 \%$ de los estudiantes considera que cuenta con estrategias suficientes frente al $12 \%$ inicial; y frente a situaciones inesperadas solo un $24 \%$ seguiría sin saber cómo reaccionar frente al $76 \%$ inicial. Sin embargo, la mejora no ha sido tan sustancial como con la autoeficacia de conocimientos, ya que se trata de estimar la competencia en situaciones impredecibles en las que pueden ocurrir muchas cosas imprevistas.

\section{CONCLUSIONES:}

Esta experiencia educativa ha contribuido a incrementar significativamente la competencia percibida y la percepción de autoeficacia, no solo en cuanto a los conocimientos y recursos que se poseen sino también respecto a las habilidades de manejo de grupos. Esta metodología se muestra útil para proporcionar práctica variada de numerosas habilidades en diferentes colectivos y con distintos objetivos en un tiempo limitado, y dicha experiencia redunda en una mayor percepción de competencia profesional que propicia una actitud de seguridad favoreciendo las habilidades de autoperfeccionamiento y el éxito en la práctica profesional.

\section{REFERENCIAS BIBLIOGRÁFICAS:}

Bandura, A. (1997). Self-efficacy: The exercise of control. New York: Freeman.

Comisión Europea (2004). Competencias clave para un aprendizaje a lo largo de la vida. Un marco de referencia europeo. Puesta en práctica del programa de trabajo Educación y Formación 2010. Disponible en http://www.educastur.princast.es/info/calidad/indicadores/doc/comision_europea.pdf

Piña, J.A. (2010). El rol del psicólogo en el ámbito de la salud: de las funciones a las competencias profesionales. Enseñanza e Investigación en Psicología, 15(2), 233-255.

Ribes, E. (2006). Competencias conductuales: Su pertinencia en la formación y práctica profesional del psicólogo. Revista Mexicana de Psicología, 23(1), 19-26.

Sánchez, M.P., Castañeiras, C.E. y Posada, M.C. (2012). Autoeficacia y autopercepción de compe- 


\section{LA FORMACIÓN DE FORMADORES EN EDUCACIÓN SEXUAL BÁSICA}

tencias para el ejercicio profesional del psicólogo. VIII Encuentro de Investigadores en Psicología del MERCOSUR, Buenos Aires.

Sanjuán, P., Pérez. A. \& Bermúdez, J. (2000). Escala de Autoeficacia General: Datos psicométricos de la adaptación para población española. Psicothema, 12, 509-513.

Stirling, M., Rees, H., Kasedde, S., \& Hankins, C. (2008) Addressing the vulnerability of young women and girls to stop the HIV epidemic in southern Africa. Ginebra: ONUSIDA.

\section{Anexo 1 \\ ESCALA DE PERCEPCIÓN DE AUTOEFICACIA Y COMPETENCIA COMO EDUCADOR SEXUAL.}

\section{Escoge la alternativa que mejor se ajuste a tu caso.}

1. Si me piden que diseñe un programa de educación afectivo-sexual para un colectivo con el que nunca he trabajado:

a) Cuento con suficientes conocimientos teóricos como para diseñar un taller dirigido a cualquier colectivo.

b) Cuento con conocimientos teóricos generales suficientes, solo tendría que buscar información específica del colectivo al que se refiere.

c) Buscaría en Internet programas semejantes ya hechos porque no sabría por dónde empezar.

2. Si me dicen que prepare un taller, escogiendo y/o creando actividades para un colectivo con el que nunca he trabajado:

a) Cuento con suficientes estrategias como para preparar actividades para cualquier colectivo.

b) Cuento con suficientes estrategias generales, solo tendría que pararme a pensar para adecuarlas al colectivo al que se refiere.

c) Buscaría en Internet talleres ya hechos porque no sabría qué actividades serían las adecuadas.

3. Si durante el curso de un taller me encuentro con alguna dificultad (ej. Alguien no quiere participar en una actividad):

a) Podría hacerle frente porque siento confianza en los recursos con los que cuento.

b) No sabría cómo reaccionar porque no tengo estrategias para manejar situaciones inesperadas

c) Al principio me bloquearía pero enseguida sabría qué hacer.

4. Si me dicen que he de trabajar con un grupo "difícill":

a) Aunque me preocupe un poco no me retraigo porque sé que cuento con estrategias para manejarlo.

b) Lo haría pero me pondría muy nervioso/a.

c) Me agobiaría tanto que no podría. 